\title{
Entropy Approach to Naphthide Systems
}

\section{Simonyan G*}

Departament of Chemistry YSU, Armenia

\section{Abstract}

It is shown that along with other geoinformation methods, one can also use the value of the Chenon entropy to assess the maturity of oil fields. It was found that in the series of naphthides natural gas $\rightarrow$ gas condensate $\rightarrow$ associated gas $\rightarrow$ oil, entropy increases, while geoecological syntropy decreases. It is curious that for natural gas $\mathrm{H}=0.41$, which indicates a high degree of freedom of the gas phase, while for oil the entropy and syntropy are approximately equal, which indicates that when oil is formed in a trap mainly from the mantle of a high-energy liquid, the structural formation organization is in equilibrium.

ISSN: 2637-8035

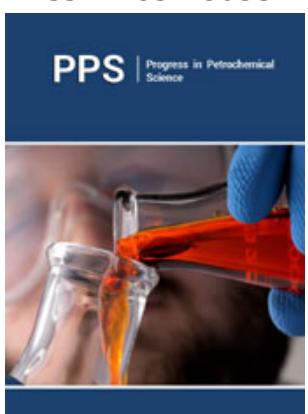

*Corresponding author: Simonyan G Departament of Chemistry YSU, Armenia sim-gev@mail.ru; gevorg.simonyan@ysu. am

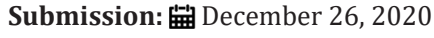

Published: 笽January 18, 2021

Volume 4 - Issue 1

How to cite this article: Simonyan Gevorg. Entropy Approach to Naphthide Systems. Progress Petrochem Sci. 4(1). PPS. 000576. 2021

DOI: $10.31031 /$ PPS.2021.04.000576

Copyright@ Simonyan G, This article is distributed under the terms of the Creative Commons Attribution 4.0 International License, which permits unrestricted use and redistribution provided that the original author and source are credited.
Keywords:Naphthides; Natural gas; Oil; Entropy; Shannon index

\section{Introduction}

Naphthides are in separate places of the Earth in gas, liquid, semi-solid and solid states or in the form of their mixture. Of greatest industrial importance are liquid naphthides called oil, or literally crude oil. Oil - complex heterogeneous colloid-dispersed systems. is formed in the interior of the Earth from deep mantle fluids and is a renewable resource. Oil is a mutual solution of the closest homologues and other compounds in each other. More than a thousand of individual organic substances were found in the composition of oil, containing: carbon, hydrogen, oxygen, nitrogen, sulfur, and more than 60 elements [1].

Gaseous naphthides are natural gas, gas condensate and associated gas [1]. Natural gas or fossil gas is a natural mixture of hydrocarbon gas, consisting mainly of methane, but usually containing various amounts of other low molecular weight alkanes, and sometimes a small percentage of carbon dioxide, nitrogen, hydrogen sulfide or helium. Natural-gas condensate, also called natural gas liquids, is a low-density mixture of hydrocarbon liquids that are present as gaseous components in the raw natural gas produced from many natural gas fields. The natural gas condensate is also called condensate, or gas condensate, or sometimes natural gasoline because it contains hydrocarbons within the gasoline boiling range. Associated petroleum gas is a natural hydrocarbon gas dissolved in oil or located in "caps" of oil and gas condensate fields. In contrast to natural gas, associated petroleum gas contains in addition to methane and ethane a large proportion of propane, butane and vapor of heavier hydrocarbons. Many associated gases, depending on the field, also contain non-hydrocarbon components: hydrogen sulfide and mercaptans, carbon dioxide, nitrogen, helium and argon.

Naphthides are unstable open geodynamic systems, which under the influence of anthropogenic, deep, surface, cosmic processes can self-organize in the direction of chaos, the measure of which is entropy or the direction of order. In open systems, which include naphthides, processes can proceed with both an increase and a decrease in entropy.

The system interacts with the outside world as a whole. An open system can exchange energy, material and, which is not less important, information with environment. The system consumes information from the environment and provides information to environment for act and interact with environment. Shannon CE [2] was the first who related concepts of entropy and information. He has suggested that entropy is the amount of information attributable to one basic message source, generating statistically independent reports. Get any amount of information entropy is equal to the lost. Information entropy for independent random event $\mathrm{x}$ with $\mathrm{N}$ possible states is calculated by the following equation:

$$
H=-\sum_{i=1}^{N} p_{i} \log _{2} p_{i}
$$

where $\mathrm{P}_{\mathrm{i}}$ is the probability of frequency of occurrence of an event. 
For the first time, in 1955, Arthur RM [3] used the general Shannon entropy equation for estimating the degree of structuring of biocenoses, in which $p_{i}$ was replaced by $p_{i}=n_{i} / N$; (where $n_{i}$ is the total number of individuals of the species $\mathrm{i}, \mathrm{N}$ is the total number of individuals in the entire biocenosis). R. Margalef postulated a theoretical concept according to which diversity corresponds to entropy when randomly choosing species from the community [4]. As a result of these works, the Shannon H Index, sometimes called the Shannon Diversity Information Index, has gained widespread acceptance.

Using the Shannon index, a comprehensive assessment of the quality of surface waters was carried out [5] and a structural analysis of the condition of Covid-19 was made [6].

The purpose of this work is to evaluate the state of naphthid systems using the chemical component composition of naphthid systems with Shannon index.

\section{Procedure for Determining}

To determine the values of the Shannon index index the following computational algorithm is used [7]:

Determines the percentage of each component-n.

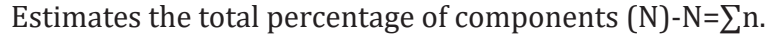

Computes $\log _{2} \mathrm{~N}, \operatorname{n\operatorname {log}_{2}} \mathrm{n}$ and $\sum \mathrm{nlog} 2 \mathrm{n}$.

Determines geoecological evolving syntropy (I) and entropy $(\mathrm{H})$ :

$$
\mathrm{I}=\sum n \log _{2} \mathrm{n} / \mathrm{N} \text { and } \mathrm{H}=\log _{2} \mathrm{~N}-\mathrm{I} .
$$

The values of $\mathrm{H}$ and I indicate what and to what extent prevails in the structure of the system: chaos or order. So, if $\mathrm{H}<\mathrm{I}$, then order prevails in the structure of the system, otherwise, when $\mathrm{H}=\mathrm{I}$ - the organization of the system is equilibrium.

\section{Result and Discussion}

Table 1: Chemical composition (vol.\%) and values of I and $\mathrm{H}$ for gas.

\begin{tabular}{|c|c|c|c|c|c|c|}
\hline \multirow[b]{2}{*}{ Composition } & \multicolumn{2}{|c|}{ Natural Gas } & \multicolumn{2}{|c|}{ Natural Gas Gas Condensate } & \multicolumn{2}{|c|}{ Associated Oil Gas } \\
\hline & $\mathbf{n}$ & nlog $\log _{2}$ & $\mathbf{n}$ & $n \log _{2} n$ & $\mathbf{n}$ & $n \log _{2} n$ \\
\hline $\mathrm{CH}_{4}$ & 94 & 615.8 & 74.8 & 465.36 & 38.8 & 204.66 \\
\hline $\mathrm{C}_{2} \mathrm{H}_{6}$ & 3 & 4.75 & 7.7 & 22.66 & 19.1 & 81.23 \\
\hline $\mathrm{C}_{3} \mathrm{H}_{8}$ & 0 & 0 & 3.9 & 7.65 & 17.8 & 73.89 \\
\hline $\mathrm{C}_{4} \mathrm{H}_{10}$ & 0 & 0 & 1.8 & 1.52 & 8 & 24 \\
\hline $\mathrm{C}_{5} \mathrm{H}_{12}$ & 0 & 0 & 6.4 & 17.13 & 6.8 & 18.79 \\
\hline $\mathrm{N}_{2}$ & 2 & 2 & 4.3 & 9.04 & 8 & 24 \\
\hline $\mathrm{CO}_{2}$ & 1 & 0 & 1.1 & 0.15 & 1.5 & 0.88 \\
\hline $\mathrm{N}$ & \multicolumn{2}{|c|}{100} & \multicolumn{2}{|c|}{100} & \multicolumn{2}{|c|}{100} \\
\hline$\sum n \log _{2} \mathrm{n}$ & \multicolumn{2}{|c|}{622.55} & \multicolumn{2}{|c|}{523.51} & \multicolumn{2}{|c|}{428} \\
\hline I & \multicolumn{2}{|c|}{6.23} & \multicolumn{2}{|c|}{5.24} & \multicolumn{2}{|c|}{4.28} \\
\hline $\mathrm{H}$ & \multicolumn{2}{|c|}{0.41} & \multicolumn{2}{|c|}{1.4} & \multicolumn{2}{|c|}{2.36} \\
\hline
\end{tabular}

In accordance with the purpose of the work and the formulation of the problem, the Shennon index of natural gas, associated petroleum gas, gas condensate fields, and oil fractions were calculated. Table 1 show the corresponding Shennon index calculations.

It is curious that for natural gas $\mathrm{H}=0.41$, which indicates a high degree of freedom of the gas phase. For oil, the Shenon entrophy $(\mathrm{H}=3.33)$ and syntropy $(\mathrm{I}=3.31)$ are approximately equal. This indicates that the structural organization of the oil is in equilibrium. Thus, it turns out that in the series of naphthides, natural gas $\rightarrow$ gas condensate $\rightarrow$ associated gas $\rightarrow$ oil, entropy increases, and geoecological syntropy decreases. Along with other geoinformation methods and technologies, it is possible to recommend using the Shennon index to assess the maturity of oil fields.

\section{References}

1. Gold T (1985) The origin of natural gas and petroleum, and the prognosis for future supplies. Ann Rev Energy 10: 53-77.

2. Shannon CE (1948) A mathematical theory of communication. Bell System Technical Journal 27(3): 379-423.

3. Arthur RM (1955) Fluctuation of animal populations and measure of community stability. Ecology 36(3): 533-536.

4. Margalef R (1958) Information theory in ecology. Gen Syst 3:36-71.

5. Simonyan G (2020) Systemic-entropic approach for assessing water quality of rivers, reservoirs, and lakes. Online First, IntechOpen, UK.

6. Simonyan GS (2020) Entropic-systems approach to assessing the structure of COVID-19. Oxidation Communications 43(4): 641-646.

7. Pirumyan GP, Simonyan GS, Margaryan LA (2019) Geoecological evaluational integrating index of natural waters and other systems. Yerevan: Copy Print LTD, Armenia, pp. 244 